\title{
A Fixed Point Theorem for Positively Curved Manifolds
}

\section{ALAN WEINSTEIN ${ }^{1}$}

\author{
Communicated by Bertram Kostant
}

1. Let $M$ be an orientable manifold of dimension $n$. If $f$ is a map from $M$ to $M$, we define the adjusted degree of $f$, denoted by adeg $(f)$, as $(-1)^{n} \operatorname{deg}(f)$. It is the contribution in dimension $n$ to the Lefschetz fixed point index of $f$. If $M$ is a Riemannian manifold, a map $f$ is called a conformal equivalence if $f$ is a diffeomorphism and $f_{*}$ is conformal on each tangent space.

The following theorem was announced in [9] in a slightly different form. Its proof is contained in Section 4.

Theorem 1. Let $M$ be a compact, connected, orientable, Riemannian manifold having strictly positive sectional curvature on all plane sections. If $f: M \rightarrow M$ is a conformal equivalence (in particular, an isometry) with adeg $(f)=1$, then $f$ has a fixed point.

In Section 2 we show that Theorem 1 implies several known theorems about positively curved manifolds. In Section 3, we discuss the conjecture that a manifold of positive curvature has positive Euler characteristic.

2. Immediate corollaries. From now on, $M$ will always be an $n$-dimensional, compact, connected, Riemannian manifold having strictly positive sectional curvature on all plane sections. Any covering space $\widetilde{M}$ of $M$ has the pulled back metric. By Myers' theorem [4], $\widetilde{M}$ is also compact.

\section{Corollary 1. (SxNGE)}

(a) If $n$ is even and $M$ is orientable, then $M$ is simply connected.

(b) If $n$ is odd, then $M$ is orientable.

Proof. We apply Theorem 1 to isometries of covering spaces of $M$. For (a), let $\tilde{M}$ be the universal covering of $M$. Any deck transformation of $\tilde{M}$ is an orientation-preserving isometry, so it has a fixed point by Theorem 1 and

\footnotetext{
${ }^{1}$ Research partially supported by an NSF Graduate Fellowship at the University of California, Berkeley.
} 
must be the identity. Since $\pi_{1}(M)$ is identifiable with the group of all deck transformations, it must be the trivial group. For (b), if $M$ is not orientable, let $\widetilde{M}$ be the orientable double covering of $M$. Then the non-trivial deck transformation of $\widetilde{M}$ has degree -1 and no fixed points, contradicting Theorem 1 .

Corollary 2. If $M$ is not orientable, then any conformal equivalence f: $M \rightarrow M$ (in particular, any isometry) has a fixed point.

Proof. By Corollary 1, $n$ is even, and the orientable double covering $\widetilde{M}$ is simply connected. $f$ lifts to $\widetilde{M}$ in two ways, one of which has degree 1 . Theorem 1 implies that this lift has a fixed point, which projects to a fixed point of $f$.

Corollary 3. If $n$ is even, any conformal vector field on $M$ (in particular, any Killing vector field) has a zero.

Proof. A nowhere-zero conformal vector field would generate fixed-pointfree conformal equivalences. If $M$ is orientable, these would have adjusted degree 1. By Theorem 1 or Corollary 2, this is impossible.

Remark. The result of Corollary 3, for Killing vector fields, has been proven directly by Berger [3].

3. The $\chi$-conjecture. It has been conjectured that if $n$ is even, $M$ must have positive Euler characteristic. If $n$ is 2 or 4, this follows from Poincaré duality and the fact that $\pi_{1}(M)$ is finite. We can push a little further with

Corollary 4. If $n=6$ and $M$ admits a non-trivial Killing vector field, then $\chi(M)>0$.

Proof. Let $X$ be a Killing vector field, not identically zero. By a theorem of Kobayashi [8], the zero set of $X$ is a disjoint union of 0-, 2-, and 4-dimensional totally geodesic submanifolds, each of which must have positive Euler characteristic. Kobayashi's theorem also states that $\chi(M)$ is the sum of the Euler characteristics of the components of the zero set. By Corollary 3, there is at least one component, so $\chi(M)>0$.

Corollary 5. If $n$ is even and $M$ admits a transitive group of isometries, then $\chi(M)>0$.

Proof. By a result of Hopf and Samelson ([7], p. 242), $M$ admits an isometry homotopic to the identity and having exactly $\chi(M)$ fixed points. Apply Theorem 1.

Remark. Corollary 5 is well known in the case that the metric on $M=G / H$ comes from a bi-invariant metric on $G$. A proof for this case is obtained by using [2], Prop. 6.1 and 6.2, then [7], p. 248.

The following conjecture, equivalent to the $x$-conjecture, would imply Theorem 1. Let $f$ be an isometry of $M$ with adeg $(f)=1$. Then the Lefschetz fixed 
point index $L(f)$ is positive. Since $\chi(M)=L$ (identity), this conjecture obviously implies the $\chi$-conjecture. Conversely, $L(f)$ is the Euler characteristic of its fixed point set, which is a non-empty union of isolated points and even-dimensional manifolds of positive curvature. (This last assertion is a slight extension of the theorem in [8]. The proof is similar to Kobayashi's, an algebraic theorem on traces replacing one on Euler characteristics.)

The only compact manifolds of positive curvature known to date are the spheres, the various projective spaces, and two odd-dimensional homogeneous spaces discovered by Berger [2]. The Lefschetz fixed point theorem can be used to show that these manifolds all satisfy the following stronger conjecture. Let $f$ be a diffeomorphism of $M$ with adeg $(f)=1$. Then $f$ has a fixed point. In fact, $L(f)>0$. It was this conjecture that led me to prove Theorem 1 for maps more general than isometries. The present method of proof seems to lead no further, though.

The last conjecture would imply that $S^{2} \times S^{2}$ has no metric of positive curvature, for the map which is the antipodal map on each factor has adjusted degree 1 and no fixed points.

The best theorem in this direction has been proven by Frankel [5], who showed that if $M$ is Kähler, then any holomorphic correspondence of $M$ has a fixed point. The importance of Frankel's result is that the class of maps to which it applies does not depend on the metric on $M$, but only on the complex structure.

Finally, in contrast to these conjectures and theorems, we should observe that nothing is known at present to disprove the possibility that every compact, simply-connected, differentiable manifold admits a metric of positive curvature.

4. Proof of Theorem 1. This proof is a combination of Frankel's idea in [5] and "Synge's trick" ([4], Thm. 11.11). Frankel has also used his method in a situation involving negative curvature [6].

Suppose $f: M \rightarrow M$ has no fixed point. The continuous function $d(x, f(x))$, where $d$ is the Riemannian distance, attains a minimum value $L>0$ at a point $p$ in $M . p$ might not be unique, so we fix a particular choice for the remainder of the proof.

Let $\alpha: R \rightarrow M$ be a geodesic parametrized by arc length with $\alpha(0)=p, \alpha(L)=$ $f(p)$. Let $e_{1}(t), \cdots, e_{n}(t)$ be an orthonormal parallel frame field along $\alpha$ such that $e_{n}(t)=\alpha^{\prime}(t)$. The map $\left(x_{1}, \cdots, x_{n}\right) \rightarrow \exp _{\alpha\left(x_{n}\right)}\left(x_{1} e_{1}\left(x_{n}\right)+\cdots+x_{n-1} e_{n-1}\left(x_{n}\right)\right)$, a diffeomorphism on a set

$$
U=\left\{\left(x_{1}, \cdots, x_{n}\right) \mid \sum_{i=1}^{n-1} x_{i}^{2}<\epsilon,-\epsilon<x_{n}<L+\epsilon\right\}, \text { for some } \epsilon>0,
$$

gives the so-called Fermi coordinates near $\alpha$. From now on, we will identify $U$ with its image in $M$. Thus, we may consider $f$ as mapping a neighborhood of $(0,0, \cdots, 0)$ into a neighborhood of $(0,0, \cdots, L)$. Also, we may form convex combinations $t \cdot x+(1-t) \cdot y$, for $x, y$ in $U$ and $0 \leqq t \leqq 1$. 
Now let $\beta$ be any geodesic with $\beta(0)=(0, \cdots, 0)$. We define the $C^{\infty}$ rectangle $\varphi(t, u)=(1-t / L) \cdot \beta(u)+(t / L) \cdot f \beta(u)$, for $0 \leqq t \leqq L$ and $u$ small. The curves $\varphi_{u}$ are defined by $\varphi_{u}(t)=\varphi(t, u) . \varphi_{0}$ is just $\alpha$. By the choice of $p$, we have $l(u)=$ length of $\varphi_{u} \geqq d(\beta(u), f \beta(u)) \geqq L$. Hence, the first and second derivatives of $l$ at $u=0$ must be zero and non-negative respectively.

To evaluate these derivatives, we first note that the transverse vector field $Y(t)=\partial \varphi /\left.\partial u\right|_{u=0}=(1-t / L) \cdot \beta^{\prime}(0)+(t / L) \cdot f_{*} \beta^{\prime}(0)$. (The euclidean structure on $U$ makes it possible to add vectors at different basepoints and to put the result at a third basepoint. In terms of the Riemannian metric, the change of basepoint is by parallel translation along $\alpha$.)

Since $\varphi_{0}$ is a geodesic, $l^{\prime}(0)$ is given by

$$
\left\langle Y(L), \varphi_{0}^{\prime}(L)\right\rangle-\left\langle Y(0), \varphi_{0}^{\prime}(0)\right\rangle=\left\langle f_{*} \beta^{\prime}(0), \alpha^{\prime}(L)\right\rangle-\left\langle\beta^{\prime}(0), \alpha^{\prime}(0)\right\rangle .
$$

Since $\beta^{\prime}(0)$ may be any tangent vector at $p$, and $l^{\prime}(0)=0$ for any $\beta$, we have $\left\langle f_{*} v, \alpha^{\prime}(L)\right\rangle=\left\langle v, \alpha^{\prime}(0)\right\rangle$ for any $v$ in $M_{p}$, the tangent space at $p$. Hence: i) if $v \perp \alpha^{\prime}(0)$, then $f_{*}(v) \perp \alpha^{\prime}(L)$; ii) $\left\langle f_{*} \alpha^{\prime}(0), \alpha^{\prime}(L)\right\rangle=1$. Now we use (for the first time!) the hypothesis that $f$ is conformal. ii) implies that $f_{*}$ is not the zero map on $M_{p}$-conformality implies that $f_{*}$ must be an isomorphism of $M_{p}$ onto $M_{f(p)}$. i) implies that $f_{*}$ takes the normal space to $\alpha^{\prime}(0)$ onto the normal space to $\alpha^{\prime}(L)$-conformality implies that $f_{*} \alpha^{\prime}(0)$ is a multiple of $\alpha^{\prime}(L)$. By ii), $f_{*} \alpha^{\prime}(0)=\alpha^{\prime}(L)$, and $f_{*}$ is actually an isometry of $M_{p}$ onto $M_{f(p)}$.

Now we go on to look at the second variation of arc length. We will always arrange to have $Y$ parallel along $\alpha$, so that

$$
\begin{aligned}
l^{\prime \prime}(0)= & \left(\left\langle\nabla_{(f \beta)},(f \beta)^{\prime}(0), \alpha^{\prime}(L)\right\rangle-\left\langle\nabla_{\beta^{\prime}} \beta^{\prime}(0), \alpha^{\prime}(0)\right\rangle\right) \\
& \left.-\int_{0}^{L}\left\langle R\left(\alpha^{\prime}, Y\right) Y, \alpha^{\prime}\right\rangle(t)\right) d t \geqq 0 .
\end{aligned}
$$

(See [1], formula for $f^{\prime \prime}(c)$ on p. 54. ( $X$ is erroneously written as $Y$ at one point in that formula). Ambrose, unlike other authors who compute the second variation, does not assume that the variation vector field is perpendicular to the geodesic.) The term involving $\nabla_{\beta^{\prime}} \beta^{\prime}$ drops out, since $\beta$ is always chosen to be a geodesic.

First we let $\beta$ be equal to $\alpha$. Then $f_{*} \beta^{\prime}(0)=\alpha^{\prime}(L)$, and

$$
Y(t)=(1-t / L) \cdot \alpha^{\prime}(0)+(t / L) \cdot \alpha^{\prime}(L)=\alpha^{\prime}(t),
$$

which is parallel along $\alpha$. Since $Y=\alpha^{\prime}, R\left(\alpha^{\prime}, Y\right)=0$, and we have

$$
l^{\prime \prime}(0)=\left\langle\nabla_{(f \alpha)}(f \alpha)^{\prime}(0), \alpha^{\prime}(L)\right\rangle \geqq 0
$$

Now let $\left(y_{1}, \cdots, y_{n}\right)$ be normal coordinates about $\alpha(L)=f(p)$ such that $\alpha^{\prime}(L)=\left.\left(\partial / \partial y_{n}\right)\right|_{y=0}$. At $y=0, g_{i j}=\delta_{i i}, \Gamma_{i j}^{k}=0$. The images under $f$ of geodesics through $p$ are curves through $f(p)$ which are geodesics for the metric $h_{i j}=\varphi g_{i j}$ induced by $f_{*}, \varphi$ being some smooth, positive function of the $y$ 's. Since $f_{*}$ is an isometry on $M_{p}, \varphi(0)=1$, and $h_{i j}(0)=\delta_{i j}$. The Christoffel sym- 
bols for $h_{i j}$ at the origin are easily computed to be $\Gamma_{i j}^{k}=\left(\frac{1}{2}\right)\left(\delta_{j k} \varphi_{i}+\delta_{k i} \varphi_{i}-\right.$ $\delta_{i j} \varphi_{k}$ ). (Subscripts on $\varphi$ denote partial differentiation by the $y^{\prime}$ s). The geodesic equations are $y_{k}^{\prime \prime}=-\sum \Gamma_{i j}^{k} y_{i}^{\prime} y_{j}^{\prime}$. For the curve $f \alpha, y_{i}^{\prime}(0)=\delta_{i n}$. By $(1), 0 \leqq$ $\left\langle\nabla(f \alpha),(f \alpha)^{\prime}(0), \alpha^{\prime}(L)\right\rangle=y_{n}^{\prime \prime}=-\Gamma_{n n}^{n}=-\left(\frac{1}{2}\right) \varphi_{n}(0)$. In the next paragraph, we will need to know about $\left\langle\nabla_{(f \beta)},(f \beta)^{\prime}(0), \alpha^{\prime}(L)\right\rangle$ when $\beta$ is a geodesic through $p$ such that for $f \beta, y_{i}^{\prime}(0)=\delta_{i 1}$. It is $y_{n}^{\prime \prime}=-\Gamma_{11}^{n}=\left(\frac{1}{2}\right) \varphi_{n}(0) \leqq 0$.

The map $f_{*}$ followed by parallel translation back along $\alpha$ is an isometry of $M_{p}$ into itself which fixes $\alpha^{\prime}(0)$. Restricted to the sphere of unit vectors perpendicular to $\alpha^{\prime}(0)$, it has the same adjusted degree as $f$. By a standard theorem of linear algebra, or by the Lefschetz fixed point theorem, or even by an inductive use of the present theorem, it has a fixed vector on this sphere. We can choose our orthonormal frame $e_{1}, \cdots, e_{n}$ so that $e_{1}(0)$ is such a vector. In the Fermi coordinates, this is $\left.\left(\partial / \partial x_{1}\right)\right|_{x=0}$, and we have

$$
\left.f_{*}\left(\partial / \partial x_{1}\right)\right|_{x=0}=\left.\left(\partial / \partial x_{1}\right)\right|_{x=(0, \cdots, L)} .
$$

If we let $\beta$ be the geodesic with initial tangent $e_{1}(0)$, the field $Y(t)$ is identically equal to $e_{1}(t)$, a parallel field along $\alpha$. Constructing a rectangle from $\beta$, we get

$$
l^{\prime \prime}(0)=\left\langle\nabla_{(f \beta)},(f \beta)^{\prime}(0), \alpha^{\prime}(L)\right\rangle-\int_{0}^{L}\left\langle R\left(\alpha^{\prime}, Y\right) Y, \alpha^{\prime}\right\rangle(t) d t .
$$

Now we use the assumption of positive curvature, which implies that the second term is strictly negative. In the previous paragraph, we proved that the first term is non-positive. Hence, $l^{\prime \prime}(0)<0$, contradicting (1).

\section{REFERENCES}

[1] W. Ambrose, The Cartan structural equations in classical Riemannian geometry, J. Ind. Math. Soc., 24 (1960) 23-76.

[2] M. Berger, Les variétés riemanniennes homogenes normales simplement connexes a courbure strictement positive, Ann. Scuola Norm. Sup. Pisa, 15 (1961) 174-246.

[3] M. Berger, Trois remarques sur les variétés riemanuiennes à courbure positive, $C . R$. Acad. Sc. Paris, 263 (1966) 76-78.

[4] R. Bishop \& R. Crittenden, Geometry of Manifolds, Academic Press, New York, 1964.

[5] T. Frankel, Manifolds with positive curvature, Pacific J. Math., 11 (1961) 165-174.

[6] T. Frankes, On theorems of Hurwitz and Bochner, J. Math. Mech., 15 (1966) 373-377.

[7] H. Hopf \& H. Samelson, Ein Satz über die Wirkungsräume geschlossener Liescher Gruppen, Comment. Math. Helv., 13 (1941) 240-251.

[8] S. Kobayashi, Fixed points of isometries, Nagoya Math. J., 13 (1958) 63-68.

[9] A. Weinstein, A fixed point theorem for positively curved manifolds. Preliminary report. Notices Amer. Math. Soc., 14 (1967) 137 (Abstract 67 T-33). 